\title{
Aggressiveness of melanoma subtypes selected by reflectance confocal microscopy
}

\author{
Marika Quadri, Alessandra Marconi, Francesca Truzzi, Roberta Lotti, Elisabetta Palazzo, \\ Francesca Farnetani, Carlo Pincelli and Giovanni Pellacani \\ Laboratory of Cutaneous Biology, Department of Surgical, Medical, Dental and Morphological Sciences,
}

University of Modena and Reggio Emilia, Modena, Italy

\begin{abstract}
Introduction
Malignant melanoma (MM) can present with different clinical, histopatological and biological aspects, suggesting an heterogeneous family of diseases rather than a unique entity (Clark et al., 1969, Zalaudek et al., 2008). In fact, $\mathrm{MM}$ is characterized by several cell populations that show diverse genotypic and phenotypic features, signalling pathway, biological behaviour and response to therapy. Traditionally, MM have been classified on the basis of growth pattern and biologic behaviour (Clark et al., 1969), but this classification is insufficient to provide enough information about causative factors or to select patients for specific treatment.

Reflectance confocal microscopy (RCM) allows to analyse architectural and cytological features at nearly histological resolution, and to visualize pigmented cell morphology better than conventional histopathology. This innovative approach offers an in vivo and in real time window into the tumor tissue when the lesion is still on patient's skin (Pupelli et al, 2013).

Pellacani and co-workers, by correlating RCM morphology with tumor and patient characteristics, have proposed the presence of four distinct melanoma subtypes (Pellacani et al., 2014). We hypothesize that RCM melanoma subtypes could present a different biological behaviour associated with a diverse degree of aggressiveness, which could also account for different response to therapy.
\end{abstract}

Objective
The aim was to study the biological
behaviour of malignant melanoma
RCM-subtypes
Materials and Methods
1) Reflectance confocal microscopy:
analysis of 85 patients with
malignant melanoma
2) Immunohistochemistry
3) NanoString technology: RNA
extracted from paraffin-embedded
melanoma tissues were analysed
for the expression of 770 genes

\section{Results}

\section{Representative Reflectance Confocal Microscopy features}

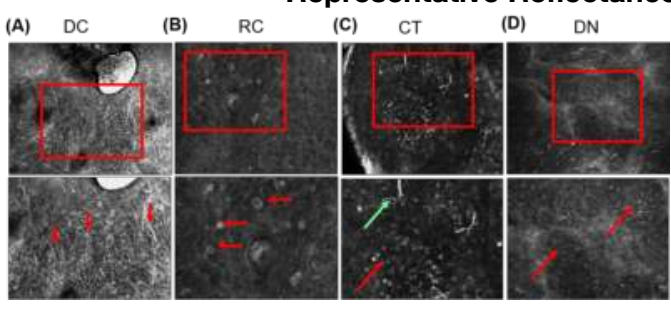

85 melanoma were retrospectively evaluated by RCM. Lesions were classified in according with their morphology in: (A) DC, for the presence of a dendritic cells population (red arrow), (B) RC, which present roundish large melanocytes with dark nuclei in the epidermidis (red arrow), (C) CT for the presence of a mixed dendric and round cells at epidermal layer, with dark nuclei (green and red arrow) and (D) DN, which presents cerebriform nesting located at dermal level (red arrow).
Association between RCM groups and patients/tumors characteristics

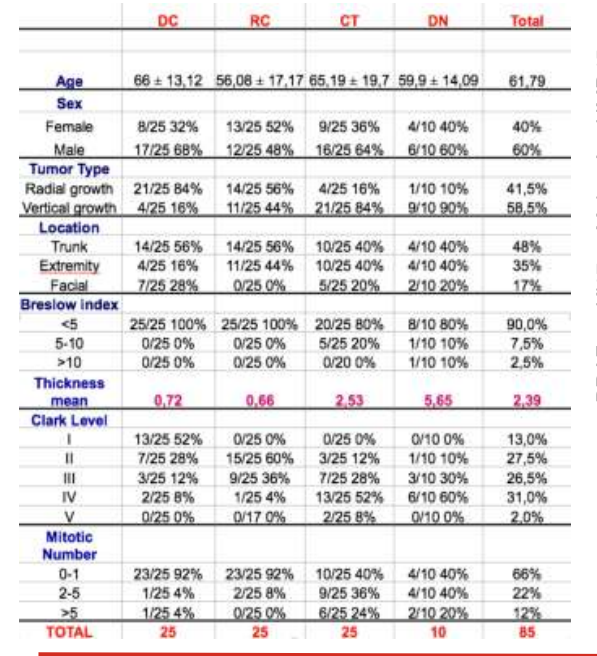

Expression of melanoma, prognostic markers and BRAF mutation DC

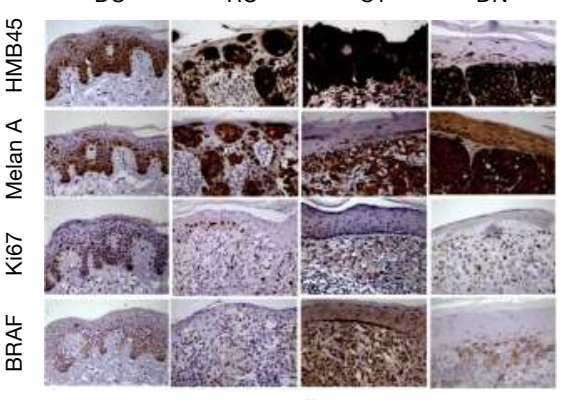

Clinical, Dermoscopic and Hystopatological features

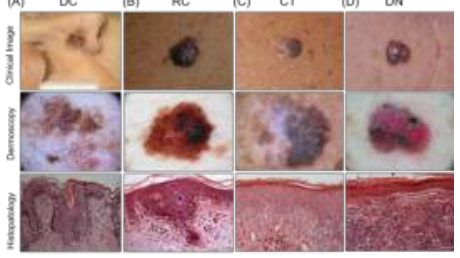

Expression of aggressivness and stemness markers

Expression was scored 0 to 4 and

the average positive cell was calculated for each individual melanoma as follow:

1) 0 score $=0 \%$

2) 1 score $=1-25 \%$

3) 2 score $=26-50 \%$

4) 3 score $=51-75 \%$

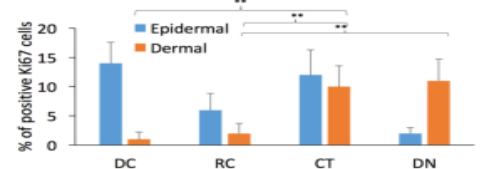

5) 4 score $=76-100 \%$ staining positive melanoma cells
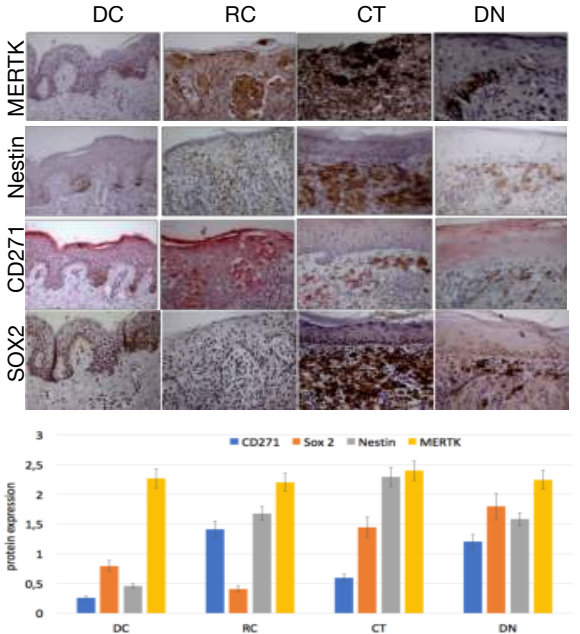

\begin{abstract}
Heatmaps of differentially expressed genes in DC, RC, CT and DN lesions Genes were clustered based on DAVID GO functional annotation tool analysis (https://david.ncifcrf.gov/).

Expression values are ranked according to their z-score after normalization. All heatmaps were generated with the $\mathrm{R}$ programming environment using Wards method with Euclidean distance (http://www.r-project.org/).
\end{abstract}
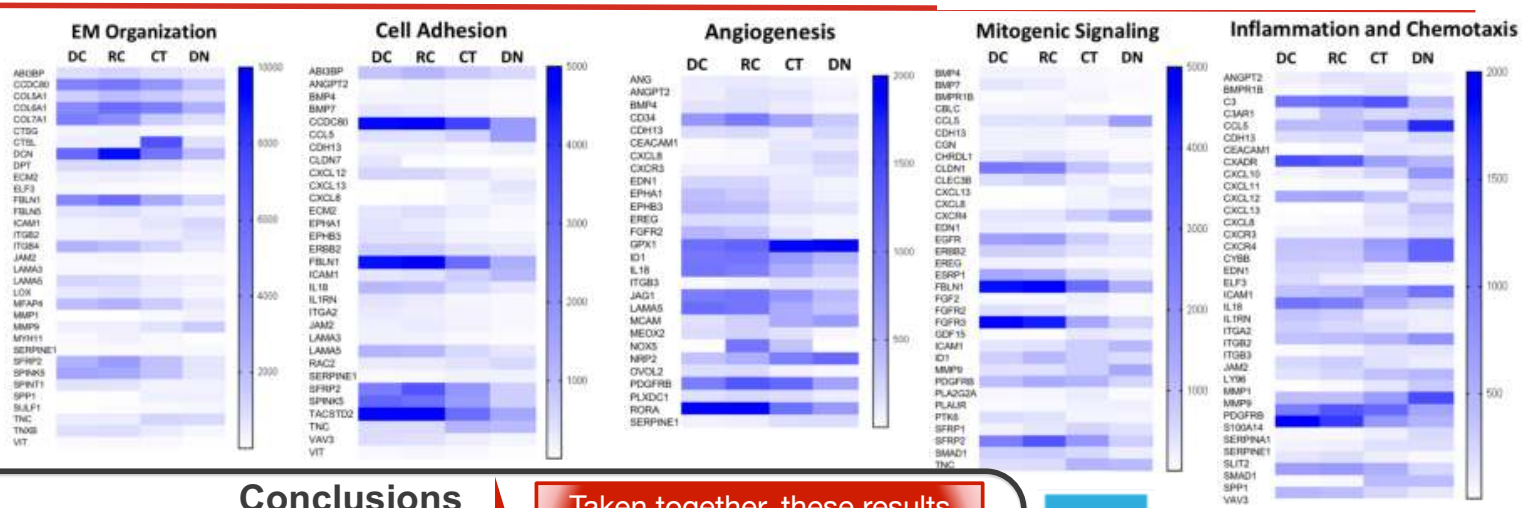

$$
\text { RC vs DC }
$$

Inflammation, chemotaxis
and migration
Angiogenesis, Response to growth factors, Cell adhesion and tissue remodeling
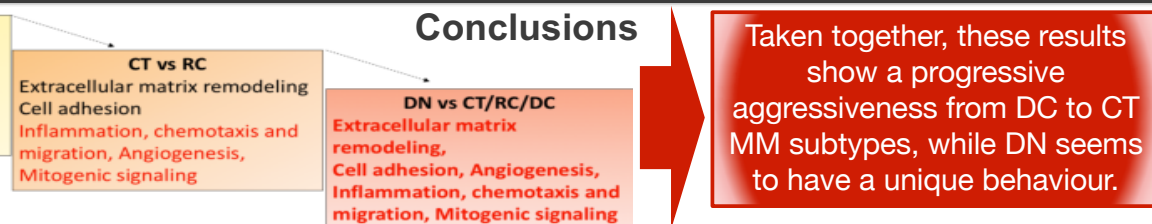